\title{
Strates
}

STRATES Matériaux pour la recherche en sciences sociales

$11 \mid 2004$

Jeune recherche, la vitalité d'un laboratoire

\section{Paysages catastrophiques au Kazakhstan}

\section{Didier Brault}

\section{OpenEdition}

Journals

Édition électronique

URL : http://journals.openedition.org/strates/426

DOI : $10.4000 /$ strates. 426

ISSN : $1777-5442$

Éditeur

Laboratoire Ladyss

Édition imprimée

Date de publication : 1 janvier 2004

ISSN : 0768-8067

\section{Référence électronique}

Didier Brault, « Paysages catastrophiques au Kazakhstan », Strates [En ligne], 11 | 2004, mis en ligne le 14 janvier 2005, consulté le 08 septembre 2020. URL : http://journals.openedition.org/strates/426 ;

DOI : https://doi.org/10.4000/strates.426

Ce document a été généré automatiquement le 8 septembre 2020

Tous droits réservés 


\title{
Paysages catastrophiques au Kazakhstan
}

\author{
Didier Brault
}

1 Quelles ont été les conditions économiques, stratégiques et culturelles qui ont abouti à créer des catastrophes écologiques durables durant la période de la colonisation russe puis l'époque soviétique? Comment ces catastrophes ont-elles généré des paysages? Telles sont les questions qui sont au centre de cette recherche. Il s'agira également d'analyser la façon dont ces paysages bouleversés sont récupérés par les médias et utilisés à des fins politiques, un paysage mythique, idéalisé, d'avant la catastrophe se trouvant transformé en un élément de la reconstruction de l'identité nationale. ${ }^{1}$

2 Par "paysages catastrophiques", j'entends des paysages où les méthodes et les conditions d'exploitation du territoire ont engendré une altération durable, tant de l'environnement que des populations qui y vivent. Ces catastrophes deviennent des paysages dans la mesure où elles fonctionnent comme modèle eschatologique : soit parce qu'elles agissent comme objet de fascination esthétique de la destruction, soit parce qu'elles permettent de développer les vertus éducatives que leur prêtent ceux qui prônent l'apprentissage de la peur quand la raison fait défaut. ${ }^{2}$

Depuis l'origine de la colonisation, outre des motifs stratégiques, la Russie a poursuivi deux buts essentiels : l'un, économique, fondé sur l'exploitation des richesses du soussol $^{3}$ et sur la mise en valeur de terres agricoles grâce à l'irrigation pour assurer son autosuffisance en fibres de coton ${ }^{4}$; l'autre, expérimental, où, compte tenu de la vacuité du territoire kazakh, l'Etat a entrepris de nombreux programmes qui ont eu un impact important sur l'aménagement du territoire, et qui l'ont conduit à en soustraire des pans entiers à des fins de sécurité. C'est dans ces espaces, à l'abri des regards étrangers, mais également de la population kazakhe, que les catastrophes ont eu lieu.

4 Le Kazakstan fut un lieu de déportation des prisonniers politiques ${ }^{5}$ et des peuples punis. Outre les camps du Goulag dans la périphérie de la ville de Karaganda ${ }^{6}$, de la fin des années 30 jusqu'au milieu de la Seconde Guerre mondiale, Staline ordonna le déplacement massif de populations de la périphérie de l'Union soviétique vers l'Asie centrale et plus particulièrement le Kazakhstan? 
5 Par ailleurs, dans le nord-est du pays fut créé le polygone d'essais nucléaire de Semipalatinsk ${ }^{8}$, dans les steppes du centre on installa la base de lancement de Baïkonour ${ }^{9}$ et une unité de recherche et de production d'armes chimiques et bactériologiques fut édifiée sur l'île de Vozkhosdenia sur la mer d'Aral. ${ }^{10}$

6 L'ensemble a nécessité des travaux colossaux : création de voies de communication et notamment de lignes de chemins de fer (le Transaralien reliant Moscou aux capitales d'Asie centrale - Almaty, Tachkent et Bichkek -, et le Turksib reliant Almaty au Transsibérien en passant par Semipalatinsk); creusement de canaux d'irrigation qui ont conduit à l'assèchement de la mer d'Aral (parmi ceux-ci, le canal du Kara Koum, sur une longueur de $1300 \mathrm{~km}$, prélève la moitié des eaux de l'Amou-Daria pour traverser les désert du Turkménistan et se diriger vers la mer Caspienne); mise en place d'une industrie lourde pour exploiter les richesses minières du sous-sol.

7 Compte tenu des richesses en minerais de fer, en charbon, plomb, zinc, uranium etc., une industrie lourde et extrêmement polluante s'est développée autour des villes de Karaganda, Oust-Kamenogorsk, Chimkent. Lors de la rupture du pacte germanosoviétique en 1941 et de l'enfoncement du front par l'armée allemande, l'Union soviétique a décidé de transporter une partie de son industrie lourde en Asie Centrale, d'où une implantation sauvage et massive qui est restée en place à l'issue de la guerre.

8 L'ensemble de ces aménagements a nécessité le déplacement de populations pour faire le vide autour de tous les sites sensibles, notamment autour des camps de relégations, des pas de tir de Baïkonour et de la zone de récupération des modules spatiaux, ainsi qu'à proximité du polygone d'essais nucléaires.

9 Tout l'aménagement du territoire fut en fait dicté par une volonté politique qui supposait de transformer en profondeur la population autochtone. La collectivisation de l'agriculture et les besoins en main-d'œuvre de l'industrie impliquaient la transformation des nomades en sédentaires - passage qui s'est effectué avec une violence effroyable et a causé la mort, par famine et par déportation, d'une part importante de la population kazakhe. ${ }^{11}$

10 La politique soviétique concernant la langue des républiques non russes conduira à une transformation tout aussi radicale. Outre le passage de l'oralité à l'écrit, il va s'agir, à partir des années 20 , de créer des langues qui correspondent au découpage territorial. De plus, l'importation massive de vocabulaire d'origine russe pour rendre compte de termes inexistants dans la langue vernaculaire va aboutir à créer des langues hybrides qui pousseront la population indigène à se russifier ${ }^{12}$. Le rattachement du Kazakhstan à l'Union soviétique supposera également le remplacement de la religion musulmane par l'idéologie communiste.

11 La mise en place du régime communiste en Asie centrale s'est accompagnée, comme dans le reste de l'Union soviétique, d'une soumission totale à l'idéologie, au travers de laquelle a été façonnée une esthétique rendant compte des transformations évoquées. À partir des années 30, les œuvres littéraires, le cinéma documentaire ou de fiction, la peinture, soumis au réalisme socialiste, les ont accompagnées et transposées. L'histoire de ces transformations du territoire ne peut s'envisager sans prendre en compte cette esthétique qui impose et justifie le rôle de l'homme soviétique vis-à-vis de son environnement. C'est de là que naît le paysage catastrophique : il est la conséquence malheureuse du paysage du démiurge. 
12 Deux sites retiendront particulièrement l'attention lors de la recherche. Le premier est celui de Semipalatinsk où la prise de conscience des intellectuels kazakhs, et notamment de Oljas Souleïmanov, au travers de la création du mouvement " Nevada Semipalatinsk », a permis, dans un climat de fin de guerre froide, d'aboutir à l'arrêt des essais nucléaires et à la reconnaissance internationale de la pollution par contamination radioactive d'une région entière et de ses effets sur la population.

La ville de Semipalatinsk se situe historiquement et géographiquement sur un point de convergence entre le Kazakhstan et la Russie. Ce sont les Russes qui ont fondé la ville où vécut plusieurs années Dostoïevski, mais c'est dans la steppe des Tchinguiz qui l'entoure que vécut Abaï Kounanbaev ${ }^{13}$. C'est donc là que se cristallisent les deux histoires, celle de l'émergence de la culture kazakhe et de sa rencontre avec la civilisation russe, et celle de sa négation au travers d'un paysage "dénaturé » où ne subsiste qu'un territoire vide. Mais c'est au travers de l'acte fondateur de l'arrêt des essais nucléaires que le président Nazerbaev a proclamé l'indépendance du Kazakhstan et si, aujourd'hui, les problèmes demeurent, les travaux de décontamination et le choix d'Abaï comme héros national contribuent à redonner un peu d'espoir quant à l'avenir de cette région.

Le second site se trouve dans la région de la mer d'Aral, et plus particulièrement sur la zone nord autour de la ville d'Aralsk et de l'embouchure du Syr-Daria ${ }^{14}$. Ville portuaire, elle est aujourd'hui en plein désert, à plus de 60 kilomètres d'un petit bout de mer. C'est également un point de rencontre des deux civilisations. La ville fut fondée par les Russes, qui construisirent des chantiers navals et une flotte de grands chalutiers, et qui peuplèrent la mer de poissons au détriment des espèces endémiques. Cette région était également l'une des voies de la Route de la soie et une terre de nomadisme où les populations vivaient soit de pêche côtière, soit de l'élevage des moutons et chameaux.

15 À l'issue de l'assèchement de la mer d'Aral, il ne reste ni mer, ni pâturage, et si les images des bateaux échoués dans le désert ont eu un grand retentissement médiatique, elles demeurent l'image d'une fatalité qui ne trouve sur place aucun début de résolution. Aujourd'hui, l'assèchement se poursuit dans la plus grande indifférence alors que les conséquences en sont multiples. Outre la disparition des poissons et donc de la pêche, il fait payer un lourd tribut à la population locale, du fait de la pollution des eaux par les produits utilisés pour la culture du coton, par la présence de sels volatiles suite à l'exondation des fonds marins et du fait de la paupérisation par l'absence de travail et donc de revenus.

16 Contrairement à la catastrophe du polygone où le paysage n'est pas transformé dans sa dimension visible ${ }^{15}$ - le territoire irradié devenu impraticable reste en apparence identique $^{16}$-, la catastrophe de la mer d'Aral a transformé physiquement le paysage. Aujourd'hui se superposent trois types de sociétés auxquelles sont associés trois paysages : celui, originel, des nomades et des bords de mer, celui de la pêche en haute mer, des bateaux et des ports où sont associés Russes et Kazakhs et qui représente pour la population d'Aralsk l'époque regrettée et enfin celui de la catastrophe où il ne reste que les carcasses des chalutiers, le fond desséché de la mer et les grues rouillées du port et où les Kazakhs se retrouvent seuls, les Russes étant partis. Tout comme sont parties les grandes administrations internationales (ONU, PNUD), aujourd'hui, onze ans après l'indépendance du Kazakhstan, il ne reste plus que quelques minuscules $\mathrm{ONG}^{17}$ et la population livrée à elle-même, au milieu du désert qui ne cesse de s'étendre. 


\section{BIBLIOGRAPHIE}

Alibek K., 2000, La guerre des germes, Presses de la cité.

Buber-Neumann M., 1986, Déporté en Sibérie, Seuil, Paris.

Imart G. et Dor R., 1982, Le chardon déchiqueté. Le nomade et le commissaire, être Kirghiz au XXe siècle, Université de Provence.

Dostoievski F. M., 1999, Les carnets de la maison morte, Actes Sud, coll. Babel.

Jonas H., 2000, Une éthique de la nature, Desclée et Bouwer.

Nekritch A., 1979, Les peuples punis, Maspéro.

Sakharov A., 1998, Les mémoires, Seuil.

Soljenitsyne A., 1968, Le pavillon des cancéreux, Livre de poche.

\section{ANNEXES}

Débat

Wanda Dressler : J'ai été intéressée par votre exposé sur le Kazakhstan, mais il m'a posé quelques problèmes. Vous parlez de la conception de la nature, et, dans le cas du Kazakhstan, de la conception de la nature russe. Il y a quand même autre chose qui s'y est appliqué : une conception du pouvoir, en relation avec ce que peut représenter le Kazakhstan pour le pouvoir russe. Voir comment les Kazakhs vivent l'aspect catastrophique de leur territoire à travers la littérature, c'est certainement un matériau très intéressant. Mais vous avez fait un collage un peu complexe, et je ne sais pas comment vous allez pouvoir théoriser tout ça.

C'est surtout le présent qui compte : comment peut-on vivre avec un territoire aussi dégradé, comment surmonter l'angoisse que cela peut créer ? Là, il y a beaucoup de questions qui se posent.

Didier Brault : Quand je parle de conception de la nature, c'est de manière très large ; ça suppose une prise en compte de tous les problèmes économiques et politiques qui vont avec, la façon dont la Russie s'est appropriée le territoire kazakh. Ce qui me paraissait intéressant - puisque j'avais fait mon DEA principalement sur la mer d'Aral - c'était de voir que l'on pouvait approcher ce problème par le côté russe (ce qui explique un peu comment, physiquement, on en est arrivé là), et puis d'un autre côté, il existe aussi l'approche, complètement exogène, de l'Occident (puisqu'en fin de compte, si tout cela a été révélé, c'est parce que cela rencontrait des soucis environnementaux « extérieurs »). Donc il y a cette double approche. L'approche de terrain sur le Kazakhstan, c'est en effet quelque chose que je n'ai pas abordé pour l'instant, si ce n'est au travers de documentaires ou de livres. C'est sûrement à creuser.

Jacques Cloarec : Je voudrais que vous m'expliquiez : qu'est-ce que le paysage vient faire là-dedans?

Didier Brault : Et bien, je pense que la prise en compte des problèmes environnementaux suppose une prise en compte « picturale » de ces problèmes : bien 
sûr, au jour le jour, les gens vivent cela de manière très concrète et très pragmatique : mais, ce que l'on peut voir aujourd'hui, c'est que le traitement de ces problèmes environnementaux se fait par toutes sortes d'organismes internationaux (notamment l'ONU) qui sont présents au travers de différentes agences (l'UNESCO, etc.). S'il y a une prise en compte internationale de ces problèmes, c'est à mon avis parce que ça fait référence à un substrat culturel, à la conception de la nature que l'on peut avoir...

Jacques Cloarec : Qui ? C'est une représentation pour qui ?

Didier Brault : Pour les Kazakhs, pour vous... justement il y en a trois : celle des Russes qui ont créé ce phénomène...

Jacques Cloarec : ... et donc qui ont produit des images paysagères sur leur action ? Il y a eu un paysage prométhéen russe : le coton, etc.

Didier Brault : Ça c'est celui de la production de films des années 20 aux années 70.

Jacques Cloarec : Vous le prenez en compte?

Didier Brault : Bien sûr, ce sera une des dimensions de l'analyse. Ensuite, il y a eu une prise en compte locale (là, je ne sais pas s'il s'agit de « paysage " pour les gens qui vivent sur place), au travers, là aussi, de documentaires, qui ont été faits après les années 90, par des Kazakhs. Et puis il y a la dimension complètement extérieure...

Jacques Cloarec : Donc vous allez étudier, comme corpus, la production documentaire des Kazakhs sur eux-mêmes, sur leur espace ? D'accord! Mais qu'est-ce qu'une figure de paysage catastrophique ? J'ai moi-même travaillé sur un petit aspect de cette question, le paysage après tempête, ou après incendie, c'est-à-dire après une catastrophe naturelle. Il faut oser penser que la catastrophe produit un paysage : quand une montagne est brûlée, comme la Sainte-Victoire, il y a un effet paysager qu'il faut oser envisager et traiter comme tel. Mais, en même temps, cela apparaît le plus souvent dans les représentations sociales comme annihilation d'un paysage, destruction, désorganisation... Ces contradictions-là, comment allez-vous les gérer?

Didier Brault : Je comprends bien le dilemme. C'est quand même une question esthétique, parce que cela renvoie à une fascination (pour la catastrophe, il y a toujours une fascination...) mais je pense que ce regard est extérieur : c'est le regard que l'on a ici, en Occident, sur ce type de paysage. Dans le cadre du DEA, sur la mer d'Aral, j'avais notamment abordé la façon dont cela avait été traité par les médias : on rencontre cette fascination à l'apparition de la catastrophe, mais très vite cela s'estompe. Justement, pour que ce soit relancé, il faut qu'il y ait une dimension esthétique, parce que les solutions n'existent pas vraiment; dans le cas de la mer d'Aral, cela fait 25 ans qu'elle a disparu et qu'il ne se passe rien. Il y a eu toutes sortes de plans échafaudés qui n'ont pas abouti ! Et la seule façon dont cela réapparaît chez nous, c'est au travers d'une image médiatique...

Jean Gardin : À propos de la mer d'Aral, un de ces bateaux perdus dans le sable était à moitié repeint : il s'appelait le Kirghiz sur la partie non repeinte, et l'Ouzbek sur la partie repeinte. Il avait été « mis en paysage ", juste sous un angle : un angle pour prendre la photo du bon côté, avec une jolie peinture et un nom local.

Wanda Dressler : Ce qui m'inquiète un peu, c'est votre approche esthétique dominante. Il faut rentrer plus dans la réalité des faits ; avec le terrain, vous verrez mieux ce qui se passe... Au niveau de la mer d'Aral, il y a des choses qui se passent, et il y a même 
quelques tentatives de résolution du problème, qui sont très marginales, mais le problème est posé.

Nicole Mathieu : Au fond, c'est vrai qu'on reste dans un compartiment un peu particulier de la recherche qui serait entre les beaux-arts et la philosophie. Si l'on essaye de sortir de ce compartiment étroit de la relation esthétique / philosophie, qui a toujours existé, je me pose cette question : pourquoi cet acharnement à vouloir absolument mettre le mot " paysage ", à nous faire entrer dans la recherche par ce mot-là ? Quand je vous entends, je me dis : au fond, quelle est la question qu'ils se posent ? Ces questions ont une relation avec ce que, nous, dans le laboratoire, nous appelons " changement social », « changement spatial ». C'est une autre façon de poser la question que de mettre, en son cœur, le changement social. Quand je vois « paysage de la routine ", je me dis que moi aussi, je rêve de faire des enquêtes sur les carrefours routiers, sur toutes sortes de formes de jardins de maisons individuelles, de pavillons, etc. , mais derrière cette question, il y a quand même, très profondément, l'idée du malêtre, du bien-être, de la domination, de l'exclusion de l'accès à certains espaces... Il y a bien d'autres choses que la question du paysage de la routine! C'est un problème tellement plus vaste... Il y a donc finalement un effacement du problème social, une focalisation sur un objet - un obscur objet du désir, mais on ne sait pas à quel désir il répond, d'ailleurs - qui empêche de poser frontalement la question qui va faire problème, et autour de laquelle on va éventuellement mobiliser le paysage, mais comme un élément, un outil, pour répondre à une démonstration.

« Paysage catastrophique » : cette question-là, elle est grave ! Quand les scientifiques oseront-ils faire une géographie ou une sociologie de la guerre, une sociologie de la domination si folle, qu'elle en arrive à nous détruire nous-mêmes? Oser le faire, ne pas tourner autour du pot par l'idée de paysage catastrophique, même si ça a une relation avec la question esthétique. Le problème, c'est quand même la catastrophe, ce n'est pas le paysage!

Donnez un questionnement qui intéresse un peu les problèmes d'aujourd'hui ! Essayons d'aller quand même aux questions dures. Même sur le " non-lieu », depuis Marc Augé qui a lancé des pistes, combien de gens ont essayé de travailler la question, en analysant les rapports que les habitants ont avec tous ces objets qui sont proposés?

Florent Hébert : Je ne pense pas que l'étude sur le paysage prétende résoudre un problème... La question n'est pas là ! En ce qui concerne ma recherche, c'est surtout raconter l'histoire d'une adaptation : voir s'il y a des points positifs... Quand on parle du lotissement, des ronds-points, on dénigre, mais, toutes les recherches...

Nicole Mathieu : Ce n'est pas l'habitant !

Florent Hébert : Ah non, ce n'est surtout pas l'habitant. Justement, je prends le problème un peu différemment. Je ne sais pas encore comment cela va se traduire, parce que je n'en suis qu'au début. Au nom du paysage, on en arrive à faire des choses suivant un regard qui n'est pas forcément adapté à une situation locale, ou sociale, ou territoriale. Donc, au nom du paysage, on peut aussi révéler qu'il existe d'autres regards, d'autres interrogations possibles. Mais, est-ce que c'est par le paysage que l'on va résoudre un problème social?

Nicole Mathieu :Je n'ai pas dit cela. Je vous ai demandé : quel est votre problème? 
Didier Brault : Le paysage, ce n'est pas une façon restrictive d'aborder un problème, c'est peut-être au contraire une façon un peu synthétique, un terme générique, qui permet de rentrer dans le sujet. Ce n'est pas pour cela que l'on s'arrête à une dimension esthétique ! C'est au contraire pour prendre le sujet de front, et le plus largement possible ! Je n'évacue absolument pas les problèmes sociologiques vis-à-vis de l'apparition des catastrophes, et pour moi, elles ne sont pas uniquement des catastrophes... Il y a obligatoirement une dimension esthétique, et c'est la façon de le présenter dans le sujet. Je ne vois pas en quoi cela évacue le problème.

Jacques Cloarec : L'esthétique est une catégorie disciplinaire comme les autres, on peut l'investir. Une problématique, ce n'est pas seulement une problématique sociale. Une problématique sociologique, ce n'est pas seulement une problématique sociale.

Nicole Mathieu : Bien sûr.

Jacques Cloarec : En même temps, la catégorie des arts, nous sommes un certain nombre à avoir essayer de démontrer depuis plusieurs années que ça s'instituait comme une vraie question de société. La vraie question est de savoir dans quelle discipline on se situe, surtout pour une thèse. Parce que, ces pauvres, on est en train de leur dire que leur boulot n'est pas intéressant !

Nicole Mathieu : Non! Je voudrais leur mettre des «banderilles » de telle manière qu'ils arrivent à sortir d'eux-mêmes! Quels sont les deux mots importants pour une recherche? « Modestie » - qu'il faut toujours avoir parce que cela ne suffit pas de s'abriter derrière Berque, Augé, etc. - et " autonomie de pensée ", c'est-à-dire construction d'une autonomie de questionnement ; travailler ce qui fait que vous avez envie de faire de la recherche, c'est extrêmement important! Si l'on reste dans la dimension esthétique, tu m'as déjà répondu : « on a contribué à démontrer que c'était une question de société ». Mais ce n'est pas ce que vous avez dit...

Jacques Cloarec : Nicole a raison de dire que l'on ne sent pas bien le questionnement qui vous habite. Florent, plus. Il a une quête, et c'est de dire : au fond, il n'y a pas de morphologie urbaine, il n'y a pas d'habiter là-dedans; il y a la recherche d'une logique de reconstitution en réseau. Mais c'est un questionnement un peu à l'aveugle, ce n'est pas encore tout à fait formulé.

Pour Didier, je pense qu'il y a un accolement arbitraire, pour l'instant, entre « paysage » et « catastrophe ». Parce que, pour une part, la catastrophe c'est un produit historique et politique très déterminé, très facile à décrire, avec ses conséquences, etc. Donc, on pourrait le faire en termes de sociologie, ou d'effet géographique d'une catastrophe politique. Le paysage ne s'impose pas comme un outil d'analyse, mais plus comme une résultante de représentations. Je rejoins Nicole sur cet aspect : on ne sent pas bien quel est votre questionnement précis. Dans une thèse, on est porté par une question qui vous mène au bout ; sans cela, on abandonne. Il faut qu'elle soit forte.

Françoise Plet : Pour ces fameux paysages de la routine, quels sont les moyens, les méthodes, que vous vous donnez pour les explorer? À travers cela, on verrait mieux, peut-être, les questions que vous vous posez.

Florent Hébert : Il y a des pistes qui sont des études plutôt locales, comparatives. On parlait de modestie tout à l'heure, je ne pense pas « m'abriter » derrière Augé : il dit des choses et l'on s'en sert, en essayant de voir ce qui est bon et pas bon. C'est vrai que depuis Non-lieux, il n'a pas beaucoup évolué : il parle du carrefour comme d'un «lieu 
d'échange ", donc n'étant pas un non-lieu. Moi, cela me fait un peu bondir, parce que carrefour = lieu d'échange, cela ne veut pas dire grand-chose. C'est pour cette raison que, pour le coup, une approche esthétique peut être intéressante ; surtout en ce moment, quand on voit ce que peuvent faire les collectivités au niveau du giratoire, tout ce jeu entre les intervenants, et, finalement, ce qu'en pense le public. C'est un peu tôt, mais je suis bien conscient qu'il y a des outils qu'il va falloir que j'adapte, et que c'est une question importante, ce " vécu ».

Jacques Cloarec : J'ai essayé de trouver, dans mon expérience, un exemple pratique de ce que vous voulez analyser : je pense à la limite de la frange d'urbanisation, à ce grignotage cancéreux du monde rural par l'extension urbaine qui ne sait plus se nommer. Au-delà de la banlieue, on ne sait plus ce que c'est. Il y a une atomisation de l'urbanisation, et il n'y a plus de dénomination. Alors, on dit « lotissement », parce que cela, on sait le repérer autour d'un village Votre zone d'investigation, elle pourrait se situer là, aux franges de l'urbanisation conquérante, où la société essaye d'anticiper sur ce qu'elle va faire, en créant ces réseaux. Je connais une route - une petite route départementale - qui quitte la zone d'urbanisation pour aller dans la Beauce. Cette route (qui dessert des petits chemins, où il $\mathrm{y}$ a, justement, des maisons individuelles qui se construisent) était, il y a dix ans encore, totalement rurale : il n'y avait pas de goudronnage, pas d'éclairage public. Maintenant, toutes les voies latérales qui vont vers les écarts sont goudronnées, il y a un éclairage public, des arrêts d'autobus, le dragage de la main-d'œuvre vers le terminus RER.

Ce que vous avez dit sur le fait que... ça circule m'a beaucoup parlé. Est-ce qu'il s'agit d'une routine, ce vécu des gens qui sont à la frange d'urbanisation? Ou est-ce que ce serait plutôt à accrocher au concept sociologique d'anomie ? C'est-à-dire : un habitat anomique ? Je prends l'exemple du giratoire. Mis à part le fait qu'on le construise en fonction de la consommation des populations (on fait un giratoire devant un Leclerc, un Champion, etc. ; il y a toute une analyse à faire aussi sur cette « mise au marché » de la population : on la « conduit »...), mis à part cela, ce que j'ai vu évoluer depuis une dizaine d'années, c'est que la fréquentation du giratoire a changé : elle est devenue agressive. Le giratoire a été fait pour éviter les carrefours francs, et l'on doit laisser la priorité à ceux qui sont dessus ; eh bien pas du tout ! Le premier qui arrive, passe... Il y a une perversion du giratoire qui s'opère.

Quand vous parlez d'une sorte de réification de l'environnement domestique immédiat, là, ce sont des catégories qu'il faudra bien analyser! Ce n'est pas Françoise Dubost et les jardins de banlieues, ce n'est plus du tout la même chose. Je pense que le terrain va vous apporter une mine de signifiants. Vous êtes sur une voie fructueuse, mais effectivement, il faut sortir du cadrage des " dinosaures », puis foncer sur le terrain et regarder comment ça se passe.

\section{NOTES}

1.Contrairement à l'Ouzbékistan.

2.Thèse défendue par Hans Jonas (2000).

3.Le Kazakhstan possède de grandes réserves de combustibles (charbon, pétrole, gaz, uranium) et de minerais (fer, nickel, plomb, etc.). 
4.À partir des années 20, l'union soviétique développa de manière intensive la monoculture du coton au détriment des cultures vivrières.

5.Voir notamment les témoignages de Margarette Bubber-Neumann dans Déporté en Sibérie (1986), où, malgré le titre, le lieu de déportation est bien le Kazakhstan, et de Soljenitsyne (1968). La déportation pour motif politique lors des purges des années 30, mais également la déportation des koulaks ont permis de fournir une main-d'œuvre nombreuse pour la réalisation des grands travaux d'infrastructure. À la fin des années 20, sept camps furent créés au Kazakhstan.

6.La déportation vers le Kazakhstan existait déjà au temps de la Russie tsariste, voir notamment le livre de Dostoievski (1999).

7.Nekritch (1979). On peut citer principalement les peuples suivants : les Polonais (36 000 en 1936 puis 110000 en 1939), les Coréens de Sibérie orientale (172 000 en 1937), les Baltes (25 000 en 1941), les Allemands de la Volga (1 200000 en 1941), les Tatars de Crimée (200 000 en 1944), les Tchétchènes du Caucase (310 000 en 1944), etc.

8.Création par décret le 21 août 1947 du polygone d'essais nucléaires de Semipalatinsk sur un territoire de $18000 \mathrm{~km}^{2}$, soit la superficie de l'Autriche. La création de ce polygone vidé de toute population n'empêchera pas la contamination des populations des villes et villages voisins (Sakharov, 1998). Il fut procédé à 550 tirs entre 1949 et 1991 dont 30 en surface, 80 en atmosphère et le reste en souterrain à partir de 1963. On estime à $300000 \mathrm{~km}^{2}$ la superficie du territoire contaminée par les effets des radiations. Le personnel qui travaillait sur le polygone habitait dans la ville de Kourtchatov, du nom du physicien père de la bombe nucléaire soviétique, ville dont l'existence fut tenue secrète jusqu'à la fin des années 80 .

9.Baïkonour se situe à l'est de la mer d'Aral sur les rives du Syr-Darya. La véritable ville de Baïkonour se situe en fait à $300 \mathrm{~km}$ au nord-est. La ville où vivait le personnel de la base de lancement spatial est Léninsk, interdite jusqu'à l'indépendance. Il a été procédé à 1600 tirs de fusées depuis le premier lancement en 1957. La récupération des morceaux de fusée ou des modules spatiaux se fait sur un territoire immense situé dans la région d'Arkalyk.

10.Soit l'île de la Résurrection. L'installation fut abandonnée en 1991 ; depuis elle est livrée au pillage, d'autant qu'avec la baisse du niveau de la mer, elle est désormais rattachée à l'ancien rivage. Un ancien scientifique kazakh, installé aux États-Unis, a relaté la teneur des expériences qui y étaient pratiquées (Alibek, 2000).

11.En 1929 Staline nomme P. I. Golochtchiokine Premier secrétaire du Parti communiste au Kazakhstan ; jusqu'en 1933 il sera le bourreau du peuple nomade et de l'élite intellectuelle kazakhe. On estime les disparitions à plus d'un million et demi de morts durant la sédentarisation des nomades kazakhs.

12.Cf. Rémy Dor et Guy Imart (1982) qui retracent l'évolution des langues centreasiatiques entre 1920 et 1980 .

13. Célèbre poète et philosophe du XIX ${ }^{\mathrm{e}}$ siècle.

14.Aralsk était un port de pêche industrielle utilisant une flotte d'une cinquantaine de chalutiers et de bateaux frigorifiques. Suite à la ponction de la totalité des eaux des deux grands fleuves qui alimentaient cette mer intérieure son niveau et son volume ont diminué de moitié. Les poissons ont totalement disparu en 1978.

15.À quelques exceptions près comme l'essai qui a consisté à créer le lac Chagan, ou comme le lieu même des explosions en surface. 
16.D'où sa dimension étrange ; de même que la ville de Pripiat en Biélorussie qui fut évacuée à la suite de l'explosion de la centrale nucléaire de Tchernobyl. Les photos montrent une ville intacte, l'étrangeté provient de l'absence de toute vie.

17.Dont une ONG danoise qui aide à l'introduction de poissons tolérant une forte salinité, notamment des carrelets. La pêche se fait désormais en barques depuis des hameaux côtiers.

\title{
RÉSUMÉS
}

Quelles ont été les conditions économiques, stratégiques et culturelles qui ont abouti à créer des catastrophes écologiques durables durant la colonisation russe, puis à l'époque soviétique, et comment ces catastrophes ont-elles généré des paysages ? Quels paysages préexistaient à ceux de la catastrophe? À l'issue de deux siècles de présence russe, comment la reconstruction d'une identité culturelle peut-elle se faire sur les séquelles de ces paysages catastrophiques?

What were the economical, strategical and cultural conditions which led to lasting ecological catastrophes during the Russian colonization and the Soviet times? How did these catastrophes generate landscapes? What landscapes existed before those of the catastrophe? After two centuries of Russian presence, how can the reconstruction of a cultural identity base itself on the legacy of those catastrophic lanscapes?

\section{INDEX}

Mots-clés : Environnement

Index géographique : Kazakhstan

\author{
AUTEUR \\ DIDIER BRAULT \\ Architecte DPLG, Géographe, doctorant au Ladyss, université de Paris 1, \\ dbrault@projetmuse.net
}

